\title{
Blister Formation on Rough and Technical Tungsten Surfaces Exposed to Deuterium Plasma
}

\author{
Armin Manhard*, Martin Balden, Udo von Toussaint \\ Max Planck Institute for Plasma Physics, Boltzmannstr. 2, 85748 Garching, Germany
}

\begin{abstract}
Up to now, blister formation on rough or technical tungsten surfaces exposed to hydrogen isotope plasma was believed to be completely suppressed. The few dedicated experiments on this issue that can be found in literature appear to support that claim. Using a novel technique of three-dimensional difference imaging of tungsten surfaces, we now demonstrate that roughness introduced by chemical etching, i.e., without the associated mechanical deformation layer introduced by grinding, only moderately reduces blistering. A technical surface with comparable roughness produced by precision grinding $\left(\mathrm{R}_{\mathrm{a}} \leq 1.6 \mu \mathrm{m}\right)$ led to a strong reduction in blister size and density, but blisters were found nevertheless. In this article we give a detailed description of the investigated rough $\mathrm{W}$ surfaces and present a statistical evaluation of blistering on these surfaces after exposure to a low-temperature deuterium plasma.
\end{abstract}

Keywords: roughness, blistering, tungsten, deuterium plasma, difference imaging, statistics

Publication information: Armin Manhard et al, Nucl. Fusion 57 (2017) 126012

DOI link: https://doi.org/10.1088/1741-4326/aa82c8

\section{Introduction}

In a prospective future nuclear fusion reactor, plasma-facing materials will be subjected to an intense flux of charged and neutral particles - predominantly hydrogen isotopes - with a distribution of kinetic energies that ranges from $\mathrm{eV}$ to $\mathrm{keV}$ depending on the location in the reactor vessel. The most intense plasma-wall interaction will take place in the divertor region, where particle energies in the $\mathrm{eV}$ range and ion fluxes up to the order of $10^{24} \mathrm{~m}^{-2} \mathrm{~s}^{-1}$ are expected. In this area, tungsten (W) is currently the most promising candidate material, since it can, e.g., withstand very high heat loads, shows no chemical reaction with hydrogen isotopes and, under the conditions mentioned above, exhibits only negligible physical sputtering. Compared to other candidate materials, the expected retention of hydrogen isotopes, specifically also tritium, is low [1], which is important for both safety and economic reasons.

\footnotetext{
* corresponding author: armin.manhard@ipp.mpg.de
} 
Most predictions for the behavior of $\mathrm{W}$ in a fusion reactor are, however, based on laboratory experiments. For such investigations, usually highly polished surfaces are used in order to reduce the complexity of the system as far as possible and be able to deduce fundamental physical processes. A common phenomenon on mirror-polished $\mathrm{W}$ surfaces exposed to hydrogen isotope - e.g., deuterium (D) - plasma is the formation of blisters, i.e., of surface features correlated with subsurface cavities filled with $\mathrm{D}_{2}$ gas [2]. Blistering has been observed in laboratory devices over a large range of parameters (see, e.g., [3, 4, 5]), and occasionally even in fusion experiments if highly polished samples were used [6]. However, most surfaces in present-day fusion experiments have a technical finish, just like plasmafacing components in a future reactor will. Up to now, the general consensus is that exposure to $\mathrm{H}$ isotope plasmas will not cause blistering on rough, technical surfaces. To the knowledge of the authors, this is mostly because blisters were never observed on such surfaces. There is also a dedicated study by Nishijima et al. investigating the effect of mechanical polishing with abrasive paper, which clearly states that this treatment eliminates blistering [7]. That article also claims that on the rough, mechanically polished sample the retention of D is reduced. A recent study by Zayachuk et al. [8] reports suppression of blistering on recrystallized $\mathrm{W}$ exposed to high-flux plasma already due to minor imperfections caused by mechanical polishing. An orientation-dependence became obvious particularly under conditions where the suppression occurred only on some of the grains.

In our present study, we re-investigated the influence of roughness and a technical surface finish in a systematic way and using novel techniques for blister detection. One key aspect of the work presented here is that a technical finish always entails a certain surface roughness in conjunction with severe mechanical damage to the $\mathrm{W}$ crystal structure near the surface. In order to separate both effects, and to produce samples with different roughness in a systematic, reproducible way, we first electropolished them to produce extremely smooth surfaces free of any damage introduced by polishing. We then chemically etched the samples for different times in order to roughen them without introducing any mechanical damage. For comparison, we also investigated a $\mathrm{W}$ sample with the as-delivered surface, i.e., with a technical surface finish. By placing " $T$ "-shaped markers on the surface using a focused ion beam (FIB) and recording carefully aligned three-dimensional (3-D) maps of reference areas around these markers using a confocal laser scanning microscope (CLSM) before and after plasma exposure, we were able to obtain difference images that clearly show blistering on all etched surfaces and even on the technical surface. We further present evidence that the mechanical deformation on the technical surface has a stronger influence on blistering than its roughness.

\section{Experiment Details}

\subsection{Sample preparation}

The base material for all W samples was obtained from PLANSEE SE (Reutte, Austria) and had a guaranteed purity of $99.97 \mathrm{wt} . \%$. It was hot rolled and subsequently ground by the manufacturer on both sides to a final thickness of $0.8 \mathrm{~mm}$. The surface was treated by 
precision grinding and specified to a roughness average of $\mathrm{R}_{\mathrm{a}} \leq 1.6 \mu \mathrm{m}$. All samples had a size of $12 \times 15 \mathrm{~mm}^{2}$ and were cut by the manufacturer from one single, larger $\mathrm{W}$ plate by electric discharge machining. We previously analyzed the microstructure of $\mathrm{W}$ material manufactured to the same specification, but from an earlier manufacturing batch [9]. There we found a dislocation density of $(3.2 \pm 1.7) \times 10^{14} \mathrm{~m} / \mathrm{m}^{3}$ derived from scanning transmission electron microscopy (STEM) and an average grain size of $1.17 \pm 0.03 \mu \mathrm{m}$ derived from image analysis of micrographs acquired with backscatter electron (BSE) contrast in a scanning electron microscope (SEM). Despite the same manufacturing specifications, we found that the current batch of samples shows a different orientation texture than the material described in [9]. Therefore, also the previously obtained dislocation density and grain size can unfortunately only serve as rough estimates for the present batch of W samples.

In our laboratory, the samples were first mechanically polished using $\mathrm{SiC}$ abrasive paper with water cooling up to P4000. After ultrasonic cleaning, they were then electropolished on all surfaces in $1.5 \mathrm{wt} . \%$ aqueous $\mathrm{NaOH}$ solution at a polishing voltage of $19.0 \mathrm{~V}$. In this state, the samples have a slightly wavy, but extremely smooth surface and show no polishing-induced deformation layer (see also [10]). We then used these "perfect" surfaces as the starting point to increase the roughness by chemical etching. For this, we used Murakami's solution, i.e., a 1:1 mixture of $10 \mathrm{wt} . \% \mathrm{KOH}$ in water and $10 \mathrm{wt} . \% \mathrm{~K}_{3}\left[\mathrm{Fe}(\mathrm{CN})_{6}\right]$ in water. This treatment ensures that the surface becomes more and more corrugated with increasing etching time, but no mechanical damage is introduced into the material. We chose etching times of 5, 15 and 30 seconds as well as 1 hour. In order to eliminate as many uncertainties as possible, we etched only half the surface of each sample and left one half in its original electropolished state. This provides a well-defined reference area directly on each sample. In addition to this model system for the effects of roughness, we also investigated one sample with the original technical surface (as described above), which in addition to its roughness also contains the mechanical deformation from grinding. This surface was only cleaned for 3 minutes in 1.5 wt. $\% \mathrm{NaOH}$ solution (without applying a voltage) to remove surface contamination, mainly due to oxidation. On that sample, no electropolished reference surface was prepared due to technical reasons. All samples where then annealed for 2 hours at $1200 \mathrm{~K}$ in vacuum $\left(\sim 10^{-6}\right.$ mbar) in order to obtain a defined annealing state for all of them, and to release hydrogen that was introduced into the $\mathrm{W}$ lattice during fabrication. According to previous experience, this kind of treatment does not affect the sample microstructure [9].

\subsection{Plasma exposure}

The samples described in section 2.1 were exposed to D plasma in the remote electron cyclotron resonance (ECR) plasma source "PlaQ" [11] (see Figure 1). It delivers 93\% of the ion flux as $\mathrm{D}_{3}{ }^{+}, 3 \%$ as $\mathrm{D}_{2}{ }^{+}$and $3 \%$ as $\mathrm{D}^{+}$, which add up to a total $\mathrm{D}$ flux of about $10^{20} \mathrm{D} / \mathrm{m}^{2} \mathrm{~s}$. The energy of the ions impacting on the samples can be adjusted by negatively biasing the sample holder to $\mathrm{V}_{\text {bias. }}$. Since $\mathrm{D}_{3}{ }^{+}$is the dominant ion, this leads to a typical energy per $\mathrm{D}$ of $E_{D}=-1 / 3 e \cdot V_{\text {bias }}+5 \mathrm{eV} / \mathrm{D}$ (e: elementary charge). The offset of $5 \mathrm{eV} / \mathrm{D}$ corresponds to the ion energy with the sample holder at floating potential and is preserved upon biasing. Recently, the total ion flux from plasma source was re-quantified for the typical operating conditions 
after some necessary technical modifications (new microwave generator and microwave coupling window). It could be shown that the total ion flux had changed only negligibly, particularly at a bias voltage of $-100 \mathrm{~V}$ [12], which was used for the work presented here. It is assumed that the ion species distribution also remained unchanged, since the operating pressure for the $\mathrm{D}_{2}$ working gas was kept at 1.0 Pa. During the original investigation [11], it was shown that the $\mathrm{D}_{2}$ gas pressure is the only parameter that noticeably influences the ion species distribution.

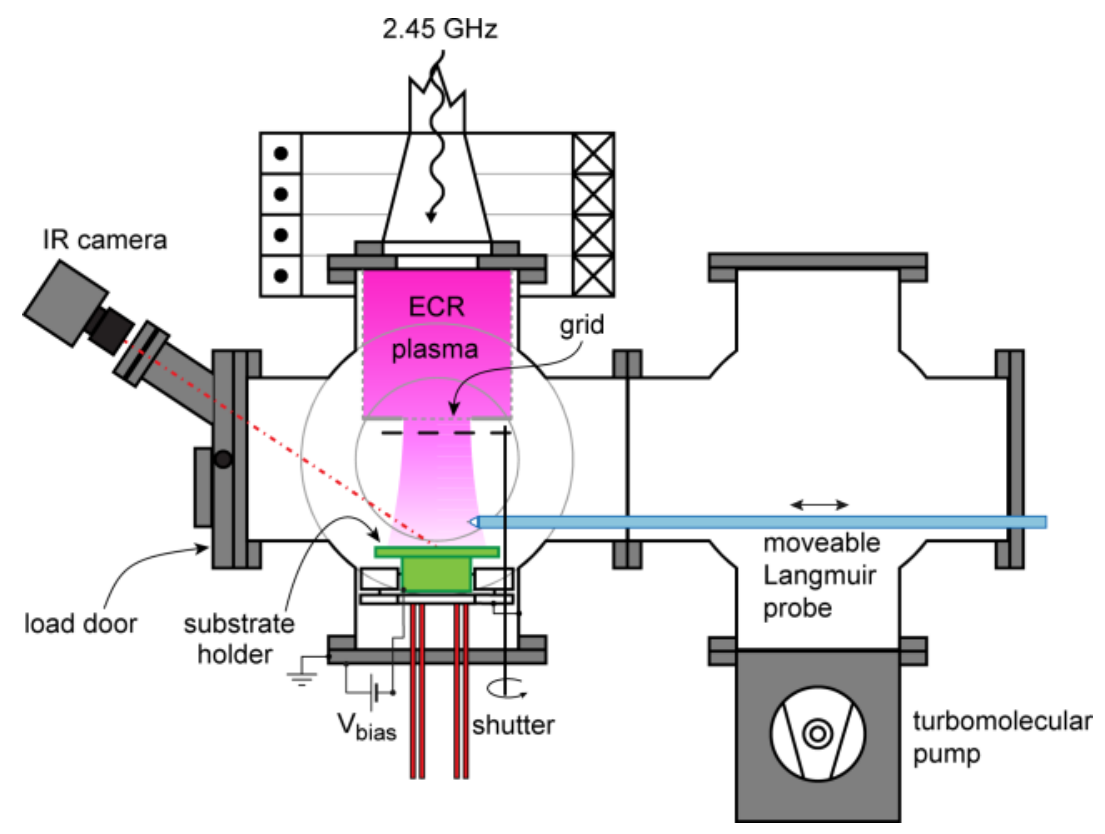

Figure 1: Schematic drawing of the plasma device "PlaQ". The figure is an updated version of the sketch shown in [11], which includes a moveable Langmuir probe for measuring the ion flux profile.

In PlaQ, multiple samples can be exposed to the plasma at once, which helps to minimize reproducibility issues when comparing different samples under the same plasma exposure conditions. On the current sample holder, which is coated with $\mathrm{W}$ by magnetron sputter deposition, up to 7 samples with a size of $12 \times 15 \mathrm{~mm}^{2}$ can be fixed using 4 molybdenum screws each. 6 samples are positioned at the same radial distance from the center, and one in the center. Since we investigated only 5 samples, all of them were mounted on the equivalent ring positions. A radial scan of the ion current was performed using a Langmuir probe biased to $-50 \mathrm{~V}$, which reciprocates $\sim 3 \mathrm{~cm}$ above the sample holder. Assuming that the measured ion current is approximately proportional to the ion flux, the radial scan indicates that the ion flux varies nearly linearly across the sample length of $15 \mathrm{~mm}$ by about $28 \%$ from the innermost to outermost edge in the radial direction. The investigated reference areas (see section 2.3) are located about $3 \mathrm{~mm}$ from the inner respectively outer edge, which reduces the flux difference between them to about $16 \%$. All samples were mounted such that the electropolished half was located closer to the sample holder center, i.e., this part of the sample received a marginally higher ion flux. The sample holder temperature is feedback controlled using a Type $\mathrm{K}$ thermocouple and a fluid thermostat. 
For our investigation, we chose a temperature of $450 \mathrm{~K}$ and a bias voltage of $-100 \mathrm{~V}$, which corresponds to $38 \mathrm{eV} / \mathrm{D}$. We exposed the samples for 66 hours, which corresponds to a fluence of about $2.4 \times 10^{25} \mathrm{D} / \mathrm{m}^{2}$. Under these conditions, the formation of numerous and large blisters can be expected on mirror-polished surfaces [5]. After plasma exposure, they were cooled to room temperature in vacuum within $\sim 15$ minutes.

\subsection{Microscopy and image processing}

Before and after plasma exposure, 3-D maps of the polished and rough sample surfaces were recorded using a confocal laser scanning microscope (CLSM; model: Olympus LEXT OLS4000). Prior to this, reference areas were marked on the roughened and polished halves of each samples by cutting 4 "T"-shaped markers with a focused ion beam (microscope: FEI HELIOS NanoLab 600). The bars of each marker have a length of $20 \mu \mathrm{m}$ and a width of 2 $\mu \mathrm{m}$. On the polished surfaces and on those etched for up to 30 seconds, the markers were cut $2 \mu \mathrm{m}$ deep. On the very rough samples ( $1 \mathrm{~h}$ etched and technical), the marker depth was increased to $4 \mu \mathrm{m}$ in order to improve marker visibility. Prior to imaging, each sample was aligned using the markers, and levelled using a gimbal-mounted sample holder until a laser scan snapshot image yielded a homogeneous illumination. Precise alignment and levelling is mandatory in order to maximize the overlap and minimize distortions between image pairs before and after plasma exposure. Images were recorded at various magnifications. Due to a large spread of relevant length scales (from sub- $\mu \mathrm{m}$ for small roughness features up to several $100 \mu \mathrm{m}$ for large blisters) and to obtain maximum height sensitivity, the most important images for analysis were composite images stitched from 5x5 3-D scans consisting of $1024 \times 1024$ pixels each. For this, a 50x objective with a numerical aperture of NA $=0.95$ was used, which yields the maximum specified height sensitivity of the microscope of $\sim 10 \mathrm{~nm}$ and a pixel size of $250 \mathrm{~nm}$. The composite images typically depict areas of approximately 1.3 $\mathrm{mm}^{2}$, which corresponds to about 21 MegaPixels per image.

For creating difference images, the rotation and lateral shift between the corresponding laser intensity images were determined using Adobe Photoshop CS 5. These images are congruent with the 3-D maps, but provide clearer contrast for the "T"-markers. In the case of the sample etched for 1 hour, also a small bi-linear distortion of the order of $0.1 \%$ was found. Image processing was subsequently performed using the Open Source software Gwyddion [13]. The 3-D maps were shifted, rotated and levelled according to the values previously determined using Photoshop. The maps acquired before and after plasma exposure were then finally cropped to their mutual intersection, levelled and subtracted from each other. In the resulting difference image, the original surface morphology is mostly removed, i.e., the background is nearly flat. Apart from a small residual background, which can be attributed to small alignment errors and imperfections in the stitching of the original composite images, only changes due to the plasma exposure (and sample handling) are visible. Particularly blisters are well recognizable due to their characteristic domed shape. As an example, the smallest blisters that were clearly recognizable in the difference image for the sample with a technical surface 
had an apex height of approximately $100 \mathrm{~nm}$. By contrast, the original 3-D map of the technical surface exhibited a surface $S_{\mathrm{q}}$ value of about $1 \mu \mathrm{m}$ (i.e., the root mean square (rms) of the heights distribution across the entire image), and height amplitudes of up to 5-6 $\mu \mathrm{m}$. This means that features with a signal to background rms ratio of less than 1:10 can be identified by this method. Further image processing, e.g., using a median filter, can further reduce the amplitudes of the residual background and somewhat improve blister visibility.

\section{Results}

\subsection{Quantitative characterization of rough surfaces}

Color-coded 3-D maps of parts of the reference areas for all investigated surfaces (after annealing) are compiled in Figure 2. In these images, you can also notice " $T$ "-shaped markers that were cut into the surface by FIB for locating the same reference area before and after plasma exposure. While the short etching times (5-30 s; Figure 2b-d) introduce increasingly deeper and more numerous pits into the originally smooth, electropolished surface (Figure 2a) due to orientation-dependent material removal, the $1 \mathrm{~h}$ treatment (Figure 2e) produces a very rough surface where only small, isolated plateaus remain unmodified. These plateau surfaces still appear very smooth and also share a common height plane. We therefore assume that they correspond to particularly chemically resistant crystal orientations that remain unaffected by the etching solution. Instead, for long etching durations, they appear to gradually shrink by chemical attack from the sides. Overall, the roughness morphology after etching appears relatively isotropic, particularly on the $1 \mathrm{~h}$ etched sample. In contrast to that, the ground, technical surface shows an extreme anisotropy: Its surface is characterized by parallel grooves and ridges that reflect the grinding direction.

When looking at the 3-D surface maps of the samples that were electropolished and etched for up to $30 \mathrm{~s}$ (Figure 2a-d), it becomes immediately apparent that the surface topography can be separated into two distinct ranges of length scales: There is a coarse-scale waviness that is characterized by wavelengths up to the $\mathrm{mm}$ range. The waviness can be seen particularly well on the electropolished surface in Figure 2a. This surface contains practically no small-scale roughness, and the only visible morphology is what we consider as waviness. In Figure $2 \mathrm{~b}-\mathrm{d}$, this waviness essentially remains, but in addition the increasing etching duration leads to the appearance of a fine-scale roughness corresponding to etching pits, which have lateral dimensions up to tens of microns. The height amplitudes of both classes of surface features are roughly comparable (see Figure $3 b$ ) - typically the amplitude of the waviness is actually somewhat larger. This means that calculation of a root mean square (i.e., $\mathrm{S}_{\mathrm{q}}$ ) roughness value for the entire surface (excluding the " $T$ " markers) is heavily influenced by the waviness component. We therefore applied a median filter to each of the surface maps (see Figure 2a-d) acquired for the samples etched for $30 \mathrm{~s}$ or less prior to plasma exposure. The waviness component is contained in the filtered image, and the roughness component is obtained by subtracting the waviness surface from the original one. It turned out that for the samples investigated here, the best radius $r_{\text {kernel }}$ for the filter kernel is about $35 \mu \mathrm{m}$ : using smaller 
kernels, the filter starts smoothing also the etch pits, and with larger kernels the filter gradually becomes unable to accurately follow the waviness profile.
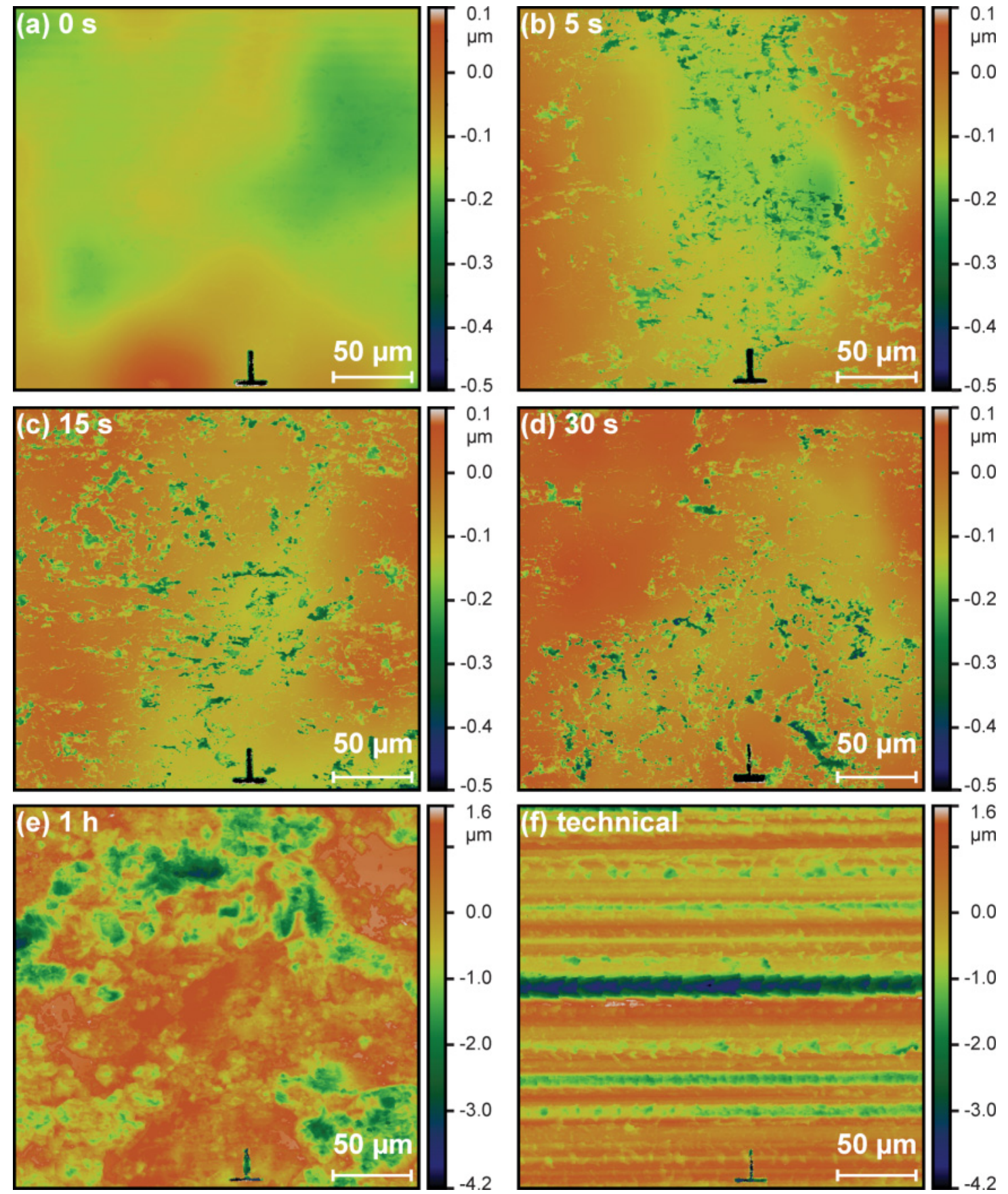

Figure 2: Color-coded 3-D surface maps of W samples with different surface roughness before plasma exposure: (a) electropolished, (b) additionally etched for $5 \mathrm{~s}$, (c) etched for 15 $s$, (d) etched for $30 \mathrm{~s}$, (e) etched for 1 hour, (f) technical surface. One of the " $T$ " shaped markers cut into the samples by FIB is visible at the bottom of each image. The image areas depicted here correspond to one single image from the 5x5 composite images that were used for data evaluation. Note that the height range in panels (e) and (f) is about 10 times larger than in panels (a)-(d). The lateral scale is identical for all panels. 
We optimized the kernel radius $r_{\text {kernel }}$ by computing the radial power spectral density functions (RPSDFs) of the original, waviness and roughness surfaces after applying median filters with different $r_{\text {kernel }}$ to a single $1024 \times 1024$ pixel sub-image of a $5 \times 5$ composite image. (The reason is that for large images and large kernel radii, the median filtering becomes very slow, which would be impractical for a parameter variation.) For small filter kernels, the low k values (i.e., large structures) were strongly suppressed in the roughness component, but particularly for $\mathrm{r}_{\text {kernel }}=10 \mu \mathrm{m}$ there was also some reduction of smaller structures (high $\mathrm{k}$ values). Increasing the filter kernel size lead to gradually weaker suppression of low $\mathrm{k}$ components, until at $\mathrm{r}_{\text {kernel }}=100 \mu \mathrm{m}$ the accuracy of the filter became clearly insufficient. The differences between results of filtering with $r_{\text {kernel }}=20,35$ or $50 \mu \mathrm{m}$ were rather small, so $r_{\text {kernel }}=35 \mu \mathrm{m}$ can indeed be seen as the best compromise. As an example for the result of median filtering with the optimal $r_{\text {kernel }}=35 \mu \mathrm{m}$, the RPSDFs corresponding to the original, waviness and roughness surfaces of the sample etched for 30 seconds are shown in Figure 3a. For this computation, we used the full $5 \times 5$ composite image. In order to further illustrate the function of our filtering method, we also show a line profile extracted from the original surface, as well as the corresponding profiles from the calculated waviness and roughness surfaces (Figure 3b). Please note that, as we described above, our calculation of the waviness surface is actually optimized with respect to the full surface maps, and not only based on a single line profile.

For the two roughest samples, i.e., the one etched for $1 \mathrm{~h}$ and the technical surface, we did not perform this scale separation into waviness and roughness. One reason is that here, the roughness component has a much larger amplitude (several $\mu \mathrm{m}$ ) than the waviness of the electropolished surface (several $100 \mathrm{~nm}$ ). Therefore, the influence of the waviness of the original surface before etching is very small. The height amplitudes on the technical surface, which is macroscopically flat by design, are comparable to those on the etched surface. The other reason is that both in the images and in the RPSDFs there is no clear separation of lateral scales, neither for the etched nor for the technical surface. We would like to note that computation of $\mathrm{S}_{\mathrm{q}}$ values described above does not follow the specification of EN ISO 25178, but is rather a practical choice based on the morphology of the investigated surfaces as well as on the technical possibilities for the data evaluation.

After applying the scale separation described above where applicable, we find that $\mathrm{S}_{\mathrm{q}}$ for the roughness component is about $5 \mathrm{~nm}$ for the electropolished surface. It increases approximately linearly up to $63 \mathrm{~nm}$ for short etching durations of 30 seconds or less. The $\mathrm{S}_{\mathrm{q}}$ value for the waviness component (not shown) scatters around $\sim 200 \mathrm{~nm}$. This is probably due to the fact that its lateral scale is comparable to the image size. For $1 \mathrm{~h}$ etching, $\mathrm{S}_{\mathrm{q}}$ reaches about $0.81 \mu \mathrm{m}$. Compared to the polished and slightly etched surfaces, the surface rms of the technical sample, $\mathrm{S}_{\mathrm{q}}=1.02 \mu \mathrm{m}$, is close to that of the $1 \mathrm{~h}$ etched sample despite its very different appearance (see Figure 4). Surface amplitudes (deepest valley to highest peak) are comparable as well and are of the order of about 5-8 $\mu \mathrm{m}$ for both samples (see Figure 2e-f and also Figure 6). 

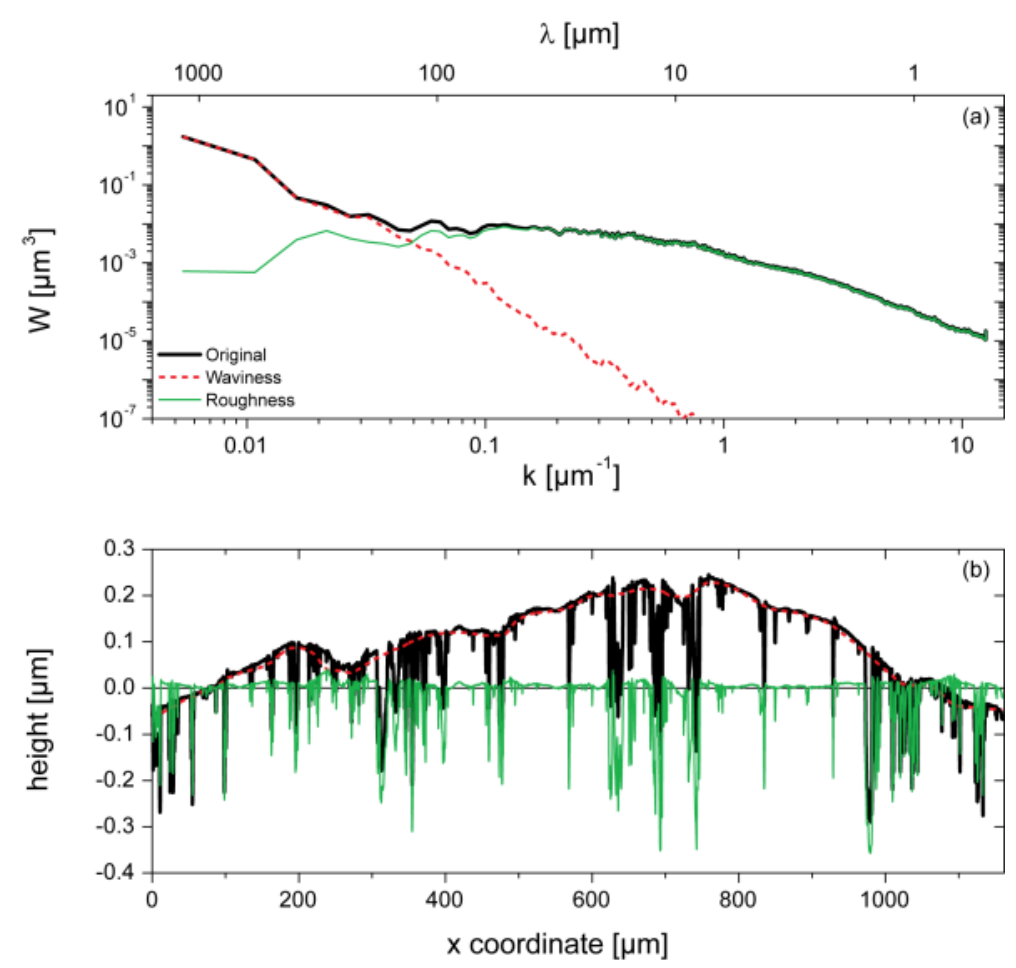

Figure 3: (a) Radial power spectral density functions of the reference area on the sample etched for 30 seconds. The solid black line represents the complete RPSDF, while the dashed red line corresponds to the waviness surface calculated by median filtering with $r_{k e r n e l}=35$ $\mu m$. The solid green line is derived from the difference image of original and waviness surface and represents the roughness component of the RPSDF. The primary $x$ axis (bottom) shows the wave number $k$ of the surface features. The secondary $x$ axis (top) indicates the corresponding length scale $\lambda=2 \pi / k$. W is the power spectral density. Panel (b) shows a line profile extracted from the original surface of this sample. The corresponding profiles extracted from the calculated waviness and roughness surfaces are also shown. The color code is identical to that in panel (a).

We also computed the surface area excess $\mathrm{X}_{\mathrm{A}}$ for all samples. We define this quantity as

$$
X_{\mathrm{A}}=\mathrm{A}_{\mathrm{eff}} / \mathrm{A}_{\mathrm{img}}-1
$$

where $A_{e f f}$ is the effective surface of the reference area, i.e., considering its 3-D topology, while $A_{i m g}$ is the projected area of the image. Since $A_{\text {eff }} / A_{\text {img }}$ is always larger than unity, we subtract 1 for clearer visualization. The surface area excess $X_{A}$ shows a similar trend as the $S_{q}$ value of the roughness component. It ranges from $2.3 \times 10^{-5}$ for the electropolished surface and peaks at 0.13 for the $1 \mathrm{~h}$ etched sample (see Figure 4 ). The technical surface exhibits only a minimally lower value of $X_{A}=0.09$. We note that the $X_{A}$ value is practically identical for the original image and for its roughness component: For the waviness surface, which was calculated for all samples etched for $30 \mathrm{~s}$ or less, $\mathrm{X}_{\mathrm{A}}$ is at least one order of magnitude smaller (of the order of $10^{-6}$ ). This clearly demonstrates that for calculating $\mathrm{X}_{\mathrm{A}}$, the separation into roughness and waviness is not necessary, since the contribution of the waviness to the total $\mathrm{X}_{\mathrm{A}}$ is negligible. 
It should be noted at this point that even for the most strongly corrugated surfaces investigated here, $\mathrm{X}_{\mathrm{A}}$ is not a particularly large quantity. Large values of $\mathrm{X}_{\mathrm{A}}$ would only be obtained for very much more extreme surface topologies, such as, e.g., tungsten fuzz created by $\mathrm{He}$ ion irradiation of $\mathrm{W}$ with high ion flux and fluence at elevated temperatures [14] or W surfaces containing deep cracks due to intense thermal shocks [15]. For comparison, a sawtooth surface consisting of triangles with an apex angle of $90^{\circ}$ would lead to $X_{A}=\sqrt{2}-1 \approx 0.41$. We would also like to note that while $\mathrm{S}_{\mathrm{q}}$ contains information about height fluctuations, $X_{\mathrm{A}}$ is linked to the distribution of inclinations $\alpha$ of surface elements with respect to an ideal plane surface (which is discussed in detail in Figure 5) via $(\cos \alpha)^{-1}$. The variation of $S_{q}$ and $X_{A}$ for the samples investigated here is summarized in Figure 4.

We analyzed the influence of our microscope's height measurement accuracy of $10 \mathrm{~nm}$ on the uncertainty of the values for $\mathrm{S}_{\mathrm{q}}$ and $\mathrm{X}_{\mathrm{A}}$ in Figure 4 and found that it is negligible due to the large number of data points. Detailed analytical derivations show that in both cases, the total uncertainty resulting from the limited accuracy of each individual data point scales like $\beta / \sqrt{N}$, where $\beta$ is of the order of the measured quantity (i.e., $\mathrm{S}_{\mathrm{q}}$ or $\mathrm{X}_{\mathrm{A}}$ ), and $\mathrm{N}$ is of the order of the number of data points per image, which is approximately $2 \times 10^{7}$. Accordingly, the relative uncertainties of $S_{q}$ and $X_{A}$ are of the order of than $0.1 \%$ or less for each of the investigated samples. We would like to stress that the values stated in Figure 4 represent in every case the entire region of interest investigated with respect to blistering (see following sections).

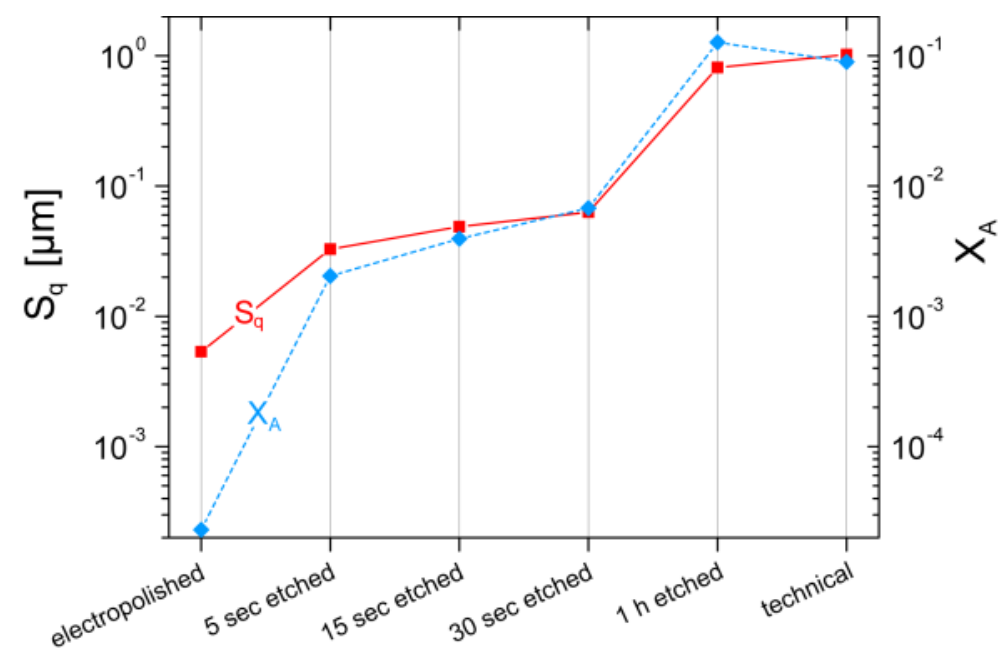

Figure 4: Surface rms $S_{q}$ (left y axis, red squares and solid line) and surface area excess $X_{A}$ (right $y$ axis, blue diamonds and dashed line) for $W$ samples with different surface treatment. Lines are only drawn to guide the eye. Please note that for the sample etched for $1 \mathrm{~h}$ and the technical surface, the original surface map was used to calculate $S_{q}$, while for the other samples only the roughness component of the surface texture was the basis for the calculation.

In principle, the parameters $\mathrm{S}_{\mathrm{q}}$ and $\mathrm{X}_{\mathrm{A}}$ neglect a large amount of information that is available about these surfaces. Substantially more detailed information can be gained from looking at 
distributions of surface parameters. E.g., the inclination distribution mentioned above (Figure 5) shows a narrow peak at $\sim 0^{\circ}$ inclination for the electropolished surface. Etching up to 30 seconds leads to an increasing tail towards larger inclinations, while the $0^{\circ}$ peak is still clearly visible. After 1 hour of etching, the inclination distribution is very broad and clearly dominated by larger inclinations. Still, a small $0^{\circ}$ peak corresponding to small, un-etched patches of the original electropolished surface remains. The inclination distribution of the technical surface is very similar to that of the $1 \mathrm{~h}$ etched sample, apart from the absence of the $0^{\circ}$ peak. The height histogram (Figure 6) shows only the roughness component for the electropolished surface and those etched for up to 30 seconds, and the full surface for the $1 \mathrm{~h}$ etched and technical surfaces. For the shorter etching durations (0-30 s), there is narrow peak corresponding to un-etched parts of the surface (which has been arbitrarily centered at $\mathrm{z}=0$ ), and a developing tail towards $\mathrm{z}<0$ that corresponds to more and deeper etch pits for increasing etching duration. The rougher samples (i.e., etched for $1 \mathrm{~h}$ and technical) show broad height histograms. As for the inclination distribution, there is a minor peak in the height histogram of the $1 \mathrm{~h}$ etched sample that originates from remaining un-etched parts of the surface. The radial power spectral density function (RPSDF, Figures 3 and 7), gives a measure of lateral length scales of the surface topography. For samples etched between 0 and 30 seconds, the RPSDF decays quickly at low k values (corresponding to length scales of $>100 \mu \mathrm{m}$ ). For these samples, this region corresponds to the waviness of the surfaces. For larger k values (i.e., smaller scales) a broad plateau follows, spanning approximately to the $1 \mu \mathrm{m}$ range and finally a decaying tail down to the lateral resolution limit. This corresponds to the roughness component. All RPSDFs for etching durations of $30 \mathrm{~s}$ or less are very similar. However, a small, systematic increase with etching duration is visible in the plateau and high $\mathrm{k}$ range (i.e., the roughness part). Both strongly roughened samples (i.e., etched for $1 \mathrm{~h}$ and technical) exhibit a broad plateau for low to intermediate k values. Interestingly, both RPSDFs are strikingly similar to each other. The oscillations in the RSPDF of the technical sample reflect the more or less regular spacing of the grooves due to grinding. For comparison, the power spectral density function (PSDF) was also computed for the $\mathrm{x}$ and $\mathrm{y}$ direction separately. For the technical surface, the PSDFs along $\mathrm{x}$ and $\mathrm{y}$ direction split on large length scales (i.e., small $\mathrm{k}$ values) such that the PSDF along the y direction closely resembles the RPSDF and also shows the same oscillations. This underlines the statement above that these features are indeed related to the grinding grooves. The directional PSDF along the $\mathrm{x}$ direction is more similar to that of the samples with short etching durations (although at a higher absolute level), i.e., the surface is comparatively smooth along the grinding grooves. For small length scales (high k), the directional PSDFs become similar to each other as well as to the RPSDF. The other samples are more or less isotropic, so the directional PSDFs are similar to the RPSDFs. Fortunately, for the surfaces investigated here, these more detailed distributions show qualitatively similar trends as the parameters $S_{q}$ and $X_{A}$. Since $X_{A}$ is, in addition, insensitive to the subtraction of the waviness surface, it is our parameter of choice for plotting trends of the samples' behavior with respect to D plasma exposure. 


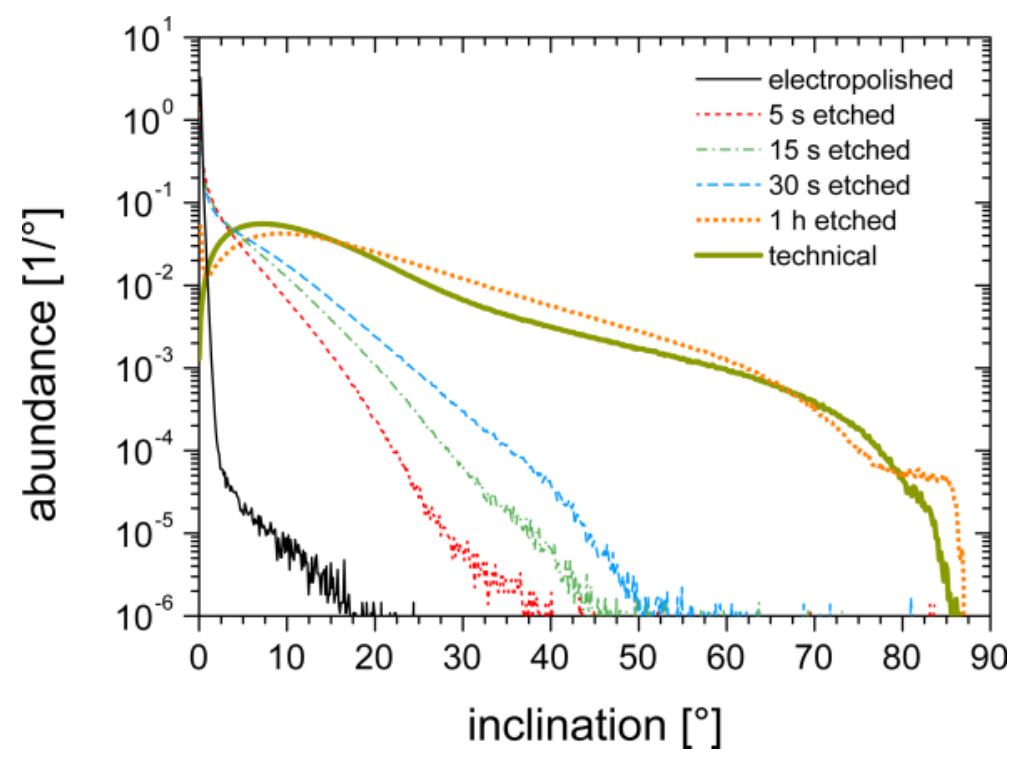

Figure 5: Inclination distributions of $W$ surfaces after different surface treatment. The full original surface map was used in the calculation. Note: The result is virtually identical if only the roughness component is used.

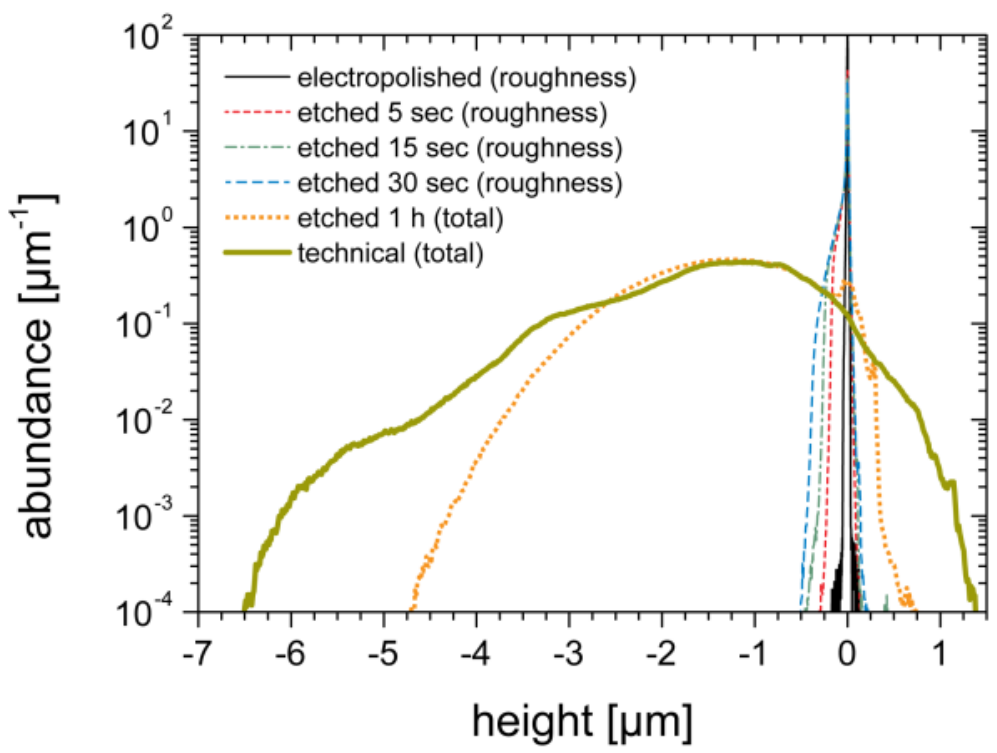

Figure 6: Height distributions of $W$ surfaces with different surface treatment. The full surface map was used for the sample etched for 1 hour and for the technical surface, for the other samples only the roughness component was evaluated. Note that the $z=0$ value is arbitrarily chosen for each curve purely on the basis of a convenient representation. 


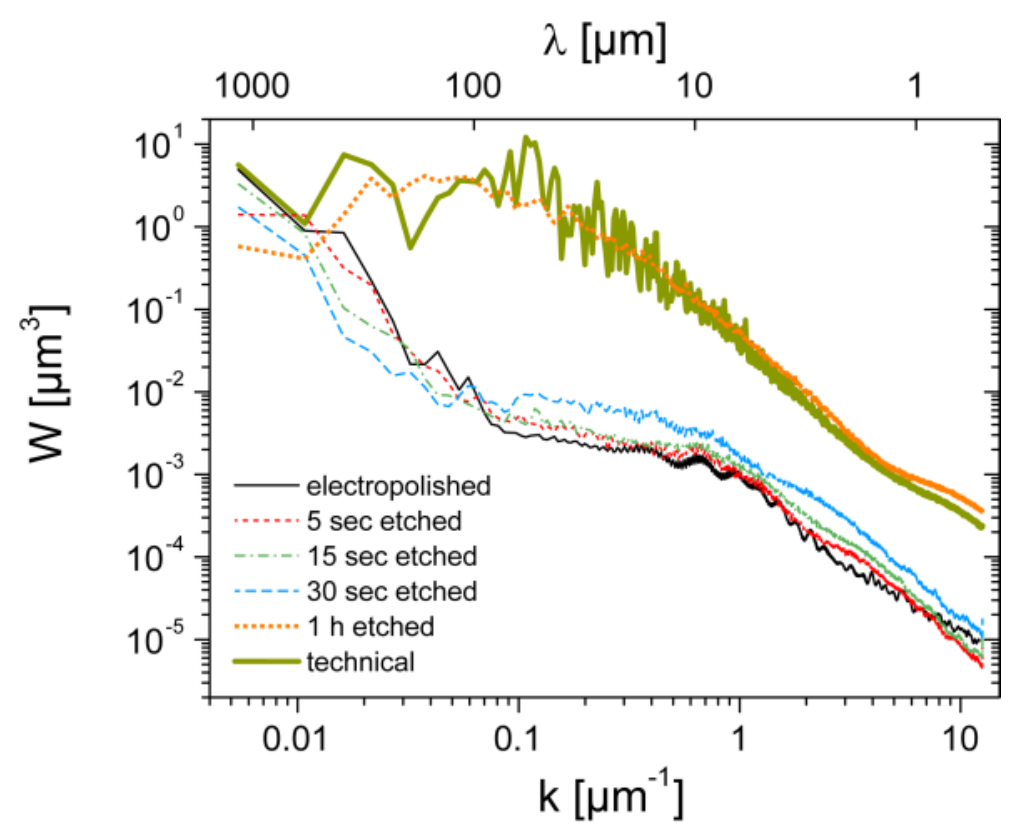

Figure 7: Radial power spectral density functions of $W$ surfaces with different surface treatments. The full surface map was used in the calculation. In contrast to the sample etched for 1 hour and the technical sample, the RPSDFs of the other samples show a "kink" around a wave number of $k=0.04 \mu^{-1}$, which is followed by a broad plateau towards larger $k$ values. This kink allows a clear distinction into waviness (small k) and roughness (large $k$ ) contributions. The primary $x$ axis (bottom) shows the wave number $k$ of the surface features. The secondary $x$ axis (top) indicates the corresponding length scale $\lambda=2 \pi / k . W$ is the power spectral density.

\subsection{Difference images of plasma-exposed samples}

For the electropolished sample as well as for the samples etched for up to 30 seconds, most blisters that were created during plasma exposure were clearly recognizable even without background subtraction. However, difference imaging makes it easier to spot also blisters with very small apex heights, and also allows more accurate blister volume measurements. For the rougher samples, i.e., the one etched for $1 \mathrm{~h}$ and the one with a technical surface, the situation is different. Without the pre-exposure reference, it is almost impossible to recognize blisters here, and only after accurate alignment and background subtraction, also the blisters with smaller apex heights become visible. In Figure 8, we demonstrate this for the example of the sample etched for $1 \mathrm{~h}$. Figure 8 a shows a laser intensity image. The rough surface can be clearly recognized, but no other features are visible. Upon careful inspection, one can notice minor differences in the 3-D maps before $(8 \mathrm{~b})$ and after $(8 \mathrm{c})$ plasma exposure that could be attributed to blistering. The difference map ( $8 \mathrm{~d}$ ) finally reveals a total of 12 large blisters with diameters up to $\sim 200 \mu \mathrm{m}$ and heights up to $\sim 3 \mu \mathrm{m}$. There is also a significant background residual with amplitudes up to about $\pm 1 \mu \mathrm{m}$ and a surface rms of about $\mathrm{S}_{\mathrm{q}} \sim 0.3 \mu \mathrm{m}$ due to the accuracy limits of the alignment. Because of this residual, it is possible that small and/or flat blisters remain undetected. Larger blisters, however, can be clearly detected on the 3-D surface maps as well as in height profiles across the surface. We would like to stress at this 
point that blister detection in optical surface images such as Fig. 8a is, as we just demonstrated, practically impossible. Typically, blisters like those shown in Fig. 8 are even harder to detect by scanning electron microscopy, since the large diameter and unfavorable aspect ratio lead to a very weak contrast already on samples without substantial roughness. This may be, in fact, the main reason why blistering on rough $\mathrm{W}$ surfaces exposed to D plasma has not been reported, or sometimes actually explicitly excluded in previous publications.
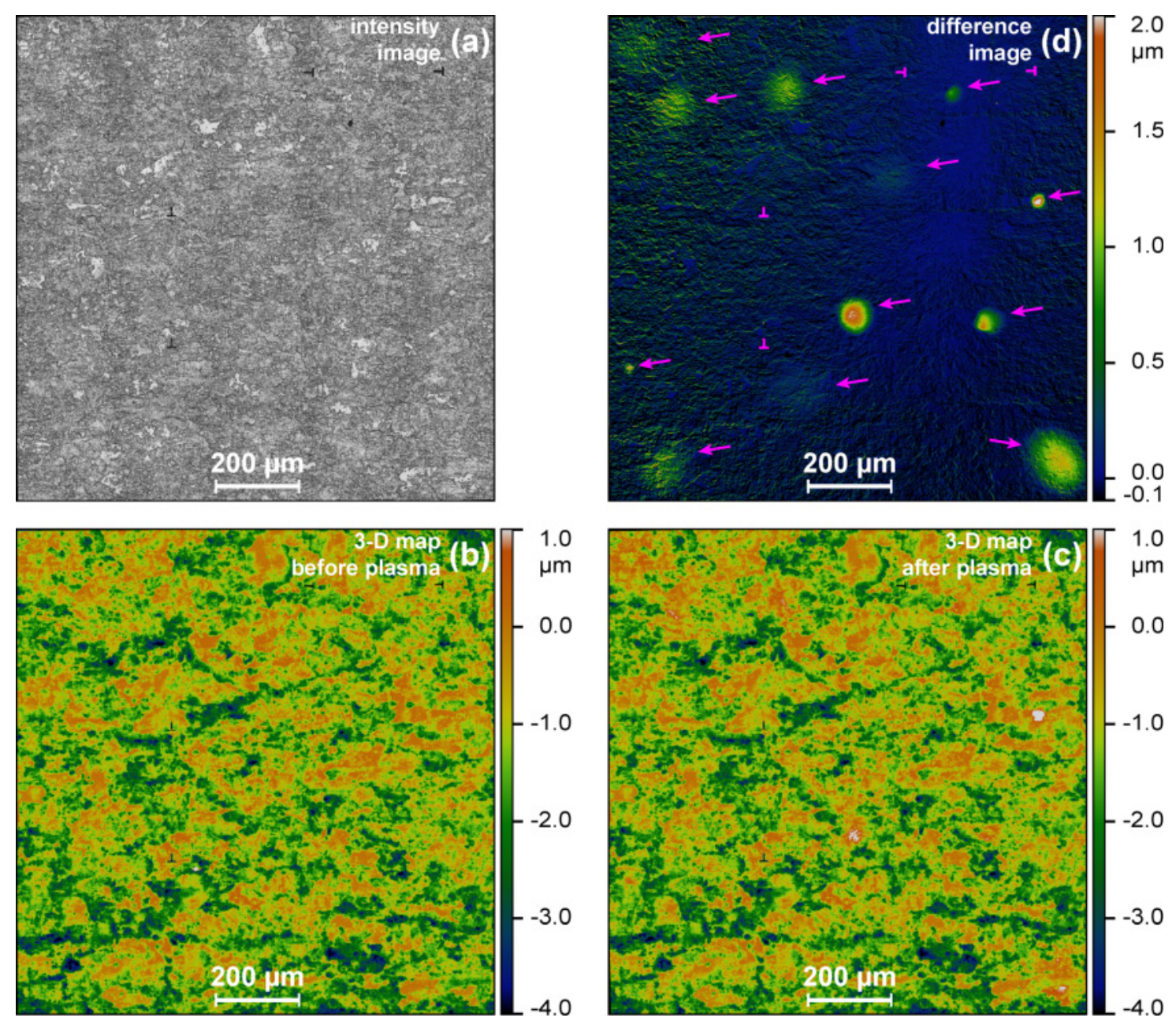

Figure 8: Demonstration of image processing in order to locate blisters on rough surfaces. Panel (a) shows a laser intensity image (i.e., an optical micrograph) of the sample etched for 1 h after plasma exposure. Blisters are not recognizable here. Panels (b) and (c) show 3-D maps of the surface before and after plasma exposure. Slight differences can be noted. Panel (d) shows the difference image (c)-(b), which clearly reveals the blisters. Note the four " $T$ "shaped markers in each image, which are marked (magenta color) in panel (d). We also added arrows indicating the positions where blisters were identified. 

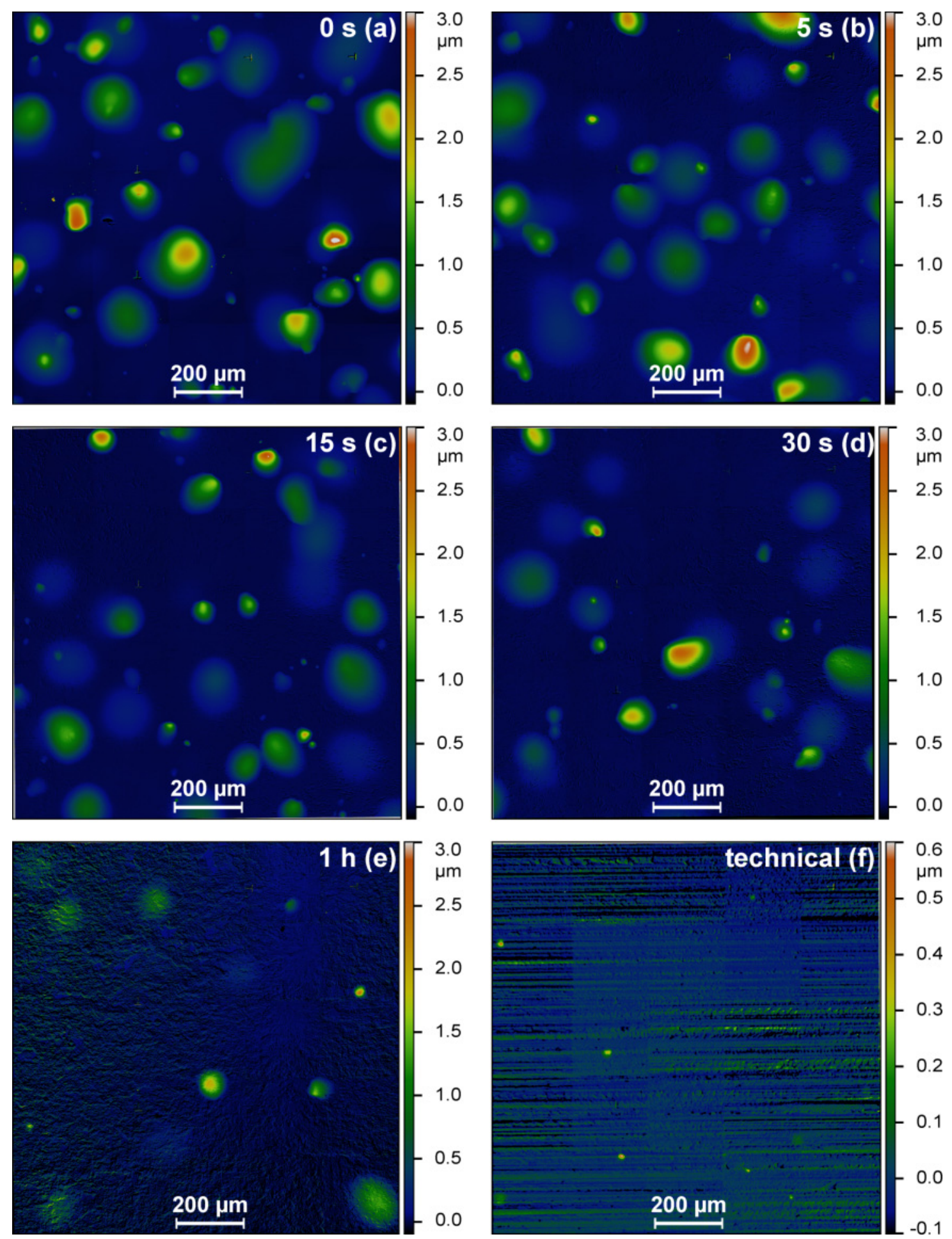

Figure 9: Difference images of tungsten surfaces with different roughness before and after plasma exposure. (a) electropolished surface, (b) etched for $5 s$, (c) etched for $15 \mathrm{~s}$, (d) etched for $30 \mathrm{~s}$, (e) etched for 1 hour, (f) technical surface. All images have the same lateral scale. The height scale is enhanced for panel (f) because of the substantially smaller size of the features. Panel (f) has been additionally post-processed using a median filter for better clarity of viewing. The original image can be found as supplemental material online. The "checkerboard" pattern (only visible in panel (f), which has the smallest height scale) is caused by small mismatches in aligning the sub-images into the $5 \times 5$ composite image. 
In Figure 9, we compiled the difference images of all investigated samples. One can immediately see that the typical size of blisters stays about constant with increasing etching duration (Figure 9a-e), while the number density gradually decreases. On the sample with a technical surface (Figure 9f), the smallest number of blisters can be found, and here also the blister size is substantially smaller than on all other samples. For improved clarity of the image, Figure 9f has also been post-processed using a median filter in order to reduce the amplitude of the residual background. (For comparison, the non-filtered difference image is provided as supplementary material). A quantitative statistical analysis of the influence of roughening and technical surface finish is presented in the following section.

\subsection{Statistical evaluation of blisters}

In order to allow a quantitative discussion of blistering on rough and technical surfaces, we computed the number density of blisters per $\mathrm{mm}^{2}$, the surface coverage $\zeta$ according to

$$
\zeta=\frac{\left(\sum_{i=1}^{N} A_{\text {blister }, i}\right)}{A_{\text {img }}}
$$

where $\mathrm{A}_{\text {blister }}$ is the projected area of a blister, $\mathrm{A}_{\mathrm{img}}$ is the projected area of the entire image and $\mathrm{N}$ is the total number of observed blisters in the image. We also calculated the average equivalent diameter $<\mathrm{d}_{\text {equiv }}>$

$$
\left\langle d_{\text {equiv }}\right\rangle=\left\langle 2 \sqrt{\frac{1}{\pi} A_{\text {blister }}}\right\rangle
$$

as well as the total blister volume per image area. These quantities are plotted versus the surface area excess $\mathrm{X}_{\mathrm{A}}$ on a logarithmic axis in Figure 10. Considering that $\mathrm{X}_{\mathrm{A}}$ varies over more than three orders of magnitude, the number density (Figure 10a) shows a surprisingly small, gradual decrease of less than one order of magnitude with increasing roughness. Following the trend line indicated in Figure 10a, one can estimate that the dependence of the number density of blisters on $\mathrm{X}_{\mathrm{A}}$ is roughly logarithmic. The sample with the technical surface shows, within the error bars, a number density of blisters that is similar to the value for the sample etched for 1 hour. Since both samples exhibit similar $\mathrm{X}_{\mathrm{A}}$ values, the sample with the technical surface in this case follows the same trend as the etched ones. . However, for all other quantities shown in Figure 10, it becomes clear that the technical surface actually behaves very different from the electropolished and etched samples. For example, the average equivalent blister diameter (Figure 10b) stays, apart from some scatter, approximately constant around 80-100 $\mu \mathrm{m}$ regardless of the etching duration, respectively of the surface area excess $X_{\mathrm{A}}$. For the technical surface, however, the average blister size is only about $30 \mu \mathrm{m}$, which is far beyond the scatter of the data for the other samples. Both surface coverage with blisters (Figure 10c) and blister volume per $\mathrm{mm}^{2}$ (Figure 10d) decrease gradually by less than a factor 10 for the etched samples with increasing $X_{A}$, again despite a variation of $X_{A}$ over more than 3 orders of magnitude. But while its $X_{A}$ value is close to that of the sample etched 
for 1 hour, surface coverage and blister volume per $\mathrm{mm}^{2}$ are more than one order of magnitude lower for the technical surface. We would like to note that this result can in principle also be derived from the product of blister density and average size: Assuming that the blister surface area is proportional to the square of the equivalent diameter, and the blister volume is to its third power, the observed trends in Figure $10 \mathrm{c}$ and $\mathrm{d}$ can be closely matched. The underlying reason is that on all investigated samples the blisters exhibit a similar domelike shape.

The error bars indicated in Figure 10 represent the statistical uncertainties of the displayed quantities. This uncertainty is due to the relatively small number of blisters within the regions of interest. For the mean equivalent diameter (Figure 10b), this uncertainty follows straightforward from the blister size distribution. For the number density of blisters (Figure 10a), we assume that the probability to detect a certain number of blisters of a given average size in a given region of interest follows a binomial distribution, and take the square root of its variance as error estimate. We further use that we can express the total blister area per image not only as the sum of all blister areas, but also as the product of number density and mean blister area. Therefore, the uncertainty of the surface coverage $\zeta$ (Figure 10c) can be estimated by error propagation of these two quantities. Estimating the uncertainty of the average blister volume per $\mathrm{mm}^{2}$ is a bit more challenging. Here we make use of the observation that all blisters roughly have a similar shape. We can therefore calculate an average proportionality constant $\langle\alpha\rangle$ between blister areas $A_{\text {blister }}$ and blister volumes $V_{\text {blister }}$ for each image, which is of the order of $20-40 \mathrm{~nm}$, so that we can approximate the total blister volume by

$$
\sum_{i=1}^{N} V_{\text {blister }, i} / A_{\text {img }} \approx\langle\alpha\rangle \cdot\left\langle A_{\text {blister }}\right\rangle \cdot \zeta(4) \text {. }
$$

We then obtain the uncertainty of the blister volume per $\mathrm{mm}^{2}$ by error propagation. We would like to point out that we actually obtained the blister volumes per $\mathrm{mm}^{2}$ shown in Figure $10 \mathrm{~d}$ via direct cumulation of the individual blister volumes in each images. Considering all the assumptions going into equation (4), the calculation described above should accordingly be seen only as a rough - but nevertheless reasonable - estimate of the actual uncertainty.

In addition to the statistical uncertainties described above, there are some inherent uncertainties of our measurement method. These would predominantly be caused by the small imperfections in alignment of the 3-D images before and after plasma exposure. Other possible source of uncertainty would be the marking of the blister outlines, as well as our currently unknown detection limit regarding small, flat blisters on noisy residual backgrounds. These are difficult to quantify accurately, but we estimate that they should be negligible compared to the statistical uncertainties: As mentioned in section 2.3, we sampled all surfaces with pixel dimensions of $250 \mathrm{~nm}$, which are far smaller than the blister size. The lateral optical resolution of the microscope, which uses a laser diode at $405 \mathrm{~nm}$ for illumination and, in our case, an objective lens with a numerical aperture of NA=0.95, is actually even better. The height uncertainty of our microscope is specified to $10 \mathrm{~nm}$ and, therefore, also much smaller than the typical height of blisters. Accordingly, we also neglect this contribution to the uncertainty of the data shown in Figure 10. 
To summarize: Varying the surface area excess $\mathrm{X}_{\mathrm{A}}$, which is a measure for the roughness, by more than 3 orders of magnitude due to chemical etching changes the statistical parameters for blistering by less than a factor 10 . On the other hand, while $\mathrm{X}_{\mathrm{A}}$ for a technical surface and a strongly etched surface differs by less than a factor 1.5, the average equivalent diameters deviate by about a factor 3 , and the surface coverages and blister volumes per $\mathrm{mm}^{2}$ lie even more than one order of magnitude apart for these two samples.
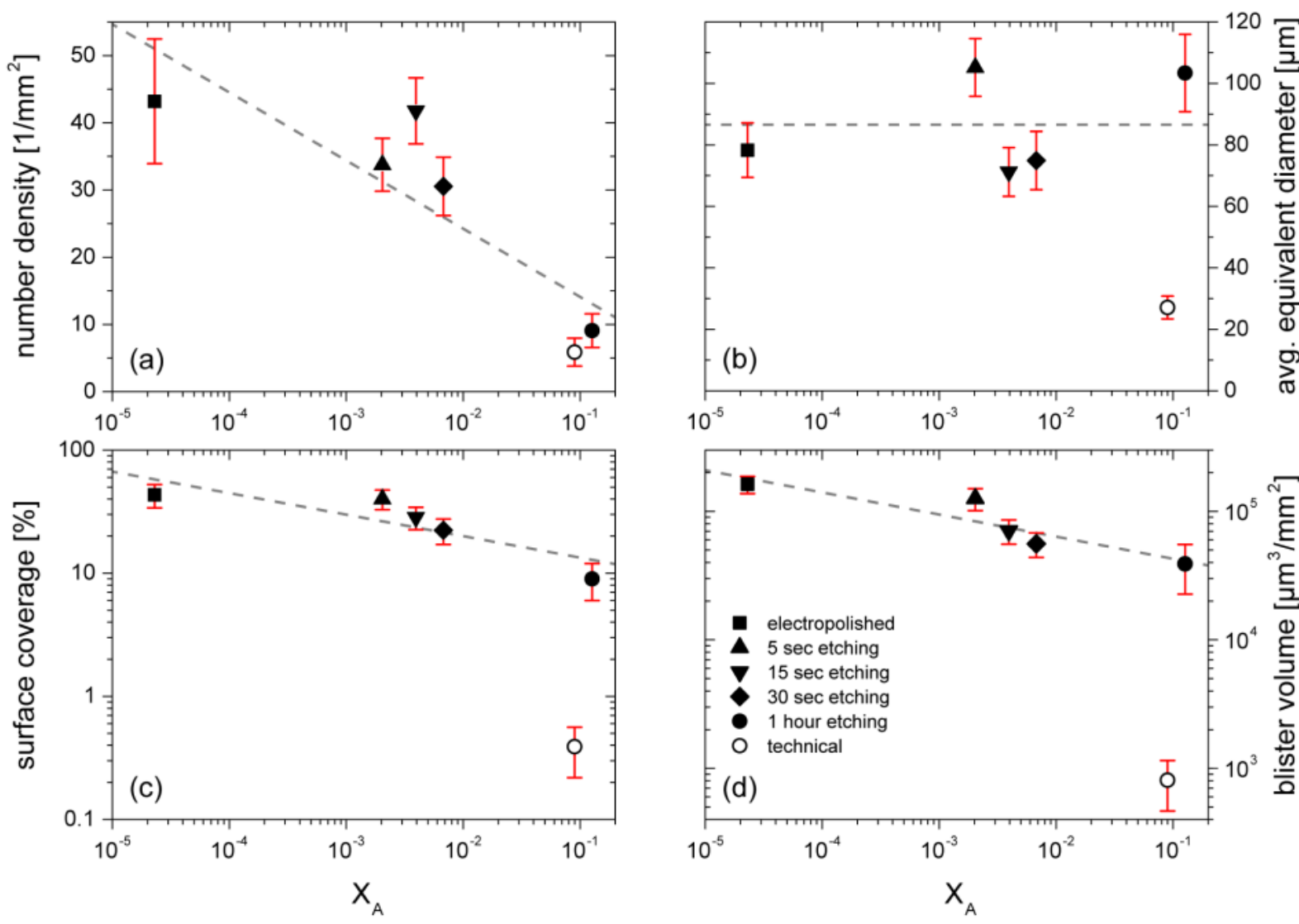

Figure 10: Statistical evaluation of the blistering behavior of $W$ samples with different surface area excess $X_{A}$. The influence of increasing roughness introduced by longer chemical etching is clearly discernible for surface coverage, number density and blister volume per surface area (filled symbols; see panels a,c and d). Etching shows no clear influence on the average blister size (panel b). Dashed lines illustrate these trends to guide the eye. The blistering behavior of the technical surface (open symbols) is in line with the trend seen for the etched samples in terms of the number density of blisters (panel a), but strongly deviates from the trend of the other samples regarding all other values.

\section{Discussion}

Looking at the results presented in section 3, the question arises which properties of rough or technical surfaces actually govern blister formation. In our discussion, we will initially focus on the electropolished and etched samples, whose only difference is the surface topography without the added complication of mechanical damage. Based on this, the results for the technical surfaces will then be discussed at the end of this section. 
From the statistical evaluation presented in section 3.3., it is clear that the roughness itself has a noticeable, systematic, but also surprisingly moderate influence. At this point, we can conceive two possible mechanisms how increasing roughness reduces blistering. First, we would like to point out that dome-shaped blisters on hot-rolled, polycrystalline $\mathrm{W}$ as we observe them here typically have their cavities located at grain boundaries parallel to the surface (see, e.g., [5, 16] and Figure 13b). One possible effect accordingly is that below a smooth, closed surface blister cavities can almost "freely" expand along grain boundaries, and their size is limited only by the D fluence, as well as by an increasing risk that the crack along the grain boundary will propagate towards the surface and the blister eventually ruptures. A rough surface, by contrast, is open and fragmented, which means that the chance that a blister cavity intersects with a free surface, even without eventually curving upward, is strongly increased. Accordingly, the blister size is either limited by the size of hills or plateaus on the surface, or the blister cavities have to be created below the deepest valley on the surface topography. To illustrate this, we have computed the distributions of height amplitudes of the rough surface before plasma exposure within the areas of the blister caps on the sample etched for 1 hour. In a typical height histogram across a single blister, we can identify the remains of the original electropolished surface as a narrow peak, which we arbitrarily center at zero elevation (see Figure 11). The actual distribution of valleys below appears as a broad peak towards negative values in this histogram.

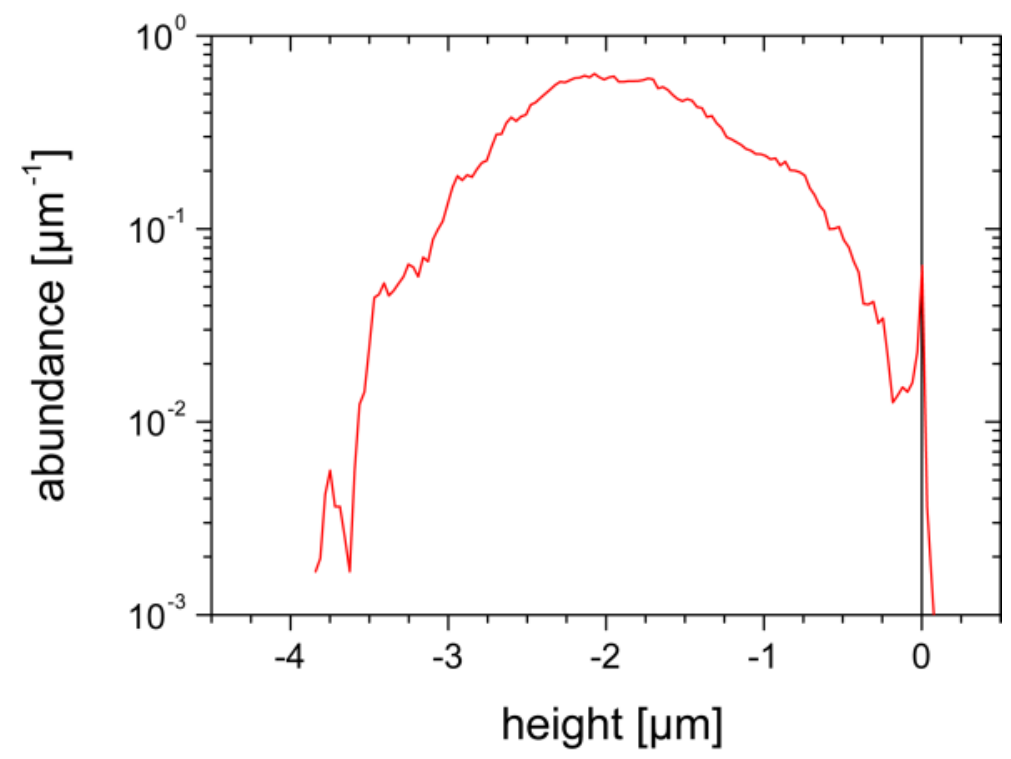

Figure 11: Height histogram across the surface corresponding to a single blister on $W$ etched for 1 hour. The histogram is taken from the original surface profile prior to plasma exposure. The value $z=0$ corresponds to the remaining islands of the original, un-etched surface.

A statistical analysis of the depth of the deepest valleys on each blister cap, i.e., of the negative cut-offs of the height histograms, reveals that the blister cavities should typically be located deeper than at least $3.5 \mu \mathrm{m}$ below the surface (see Figure 12). This effectively reduces the number of locations where large, dome-shaped blisters as we observe them here can grow. 


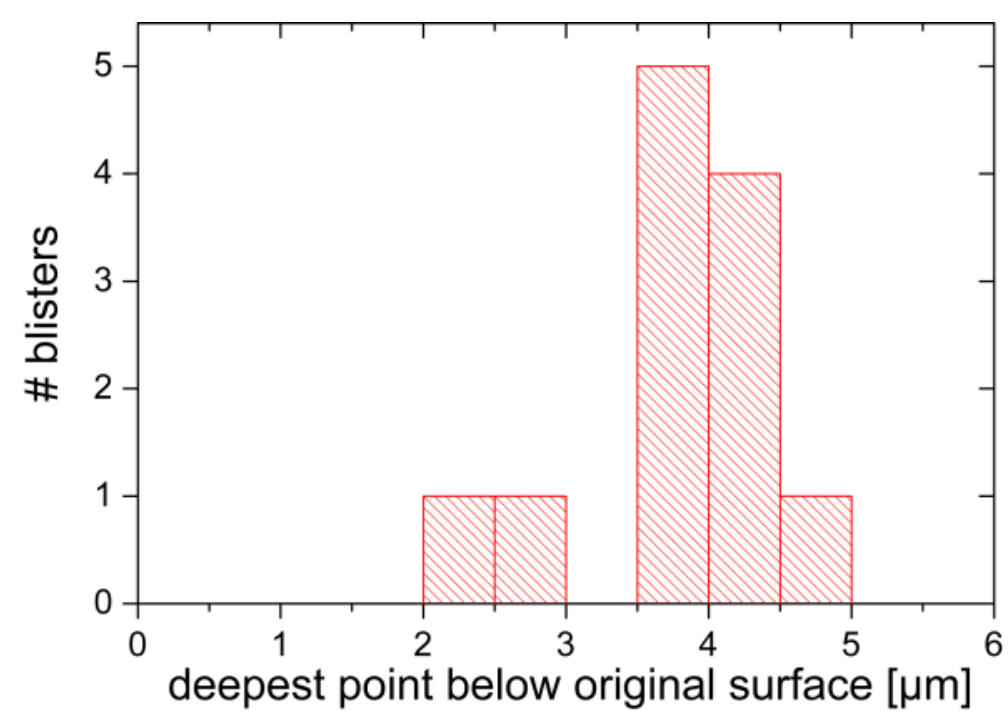

Figure 12: Histogram of differences between original flat surface and deepest point on the unexposed surface for each blister on the W sample etched for 1 hour. This histogram is based on the height histograms for the individual blisters, as shown in Figure 11.

Figure 13 shows an explicit example where a cross-section cut was prepared by focused ion beam (FIB) through one of the blisters on the sample etched for 1 hour. Figure 13a shows a detail roughly from the center of Figure 8c (note the "T"-marker in the lower left corner). The blister circumference is outlined on this image based on the difference image in Figure 8d. The additional dashed black line indicates the location of the FIB cut. One can clearly see from the SEM image of the cross-section prepared by FIB in Figure 13b that the actual cavity runs approximately parallel to the grain boundaries, which are in turn parallel to the original, electropolished surface. The largest thickness of the blister cap is of the order of $10 \mu \mathrm{m}$. At this position, the blister cavity is located even substantially deeper than the deepest valley within this cross-section, which coincidentally is also one of the deepest valleys on the entire blister cap. This illustrates that our estimate based on the height histogram across a blister cap (Figure 12) indeed delivers a lower bound for the cap thickness. Under the conditions investigated here, cavities can actually be located substantially deeper than this estimate. It is also interesting to note that the center of the blister cavity is in this case located approximately at the thickest point of the cap. From this we conclude that blister nucleation on rough samples is not necessarily restricted to deep valleys, but can also occur under "hills". From there on, the lateral growth of the cavity is then potentially restricted by intersection with neighboring valleys, particularly if the cavity curves upwards at its edges, as it is visible on the left hand side of Figure 13b. 


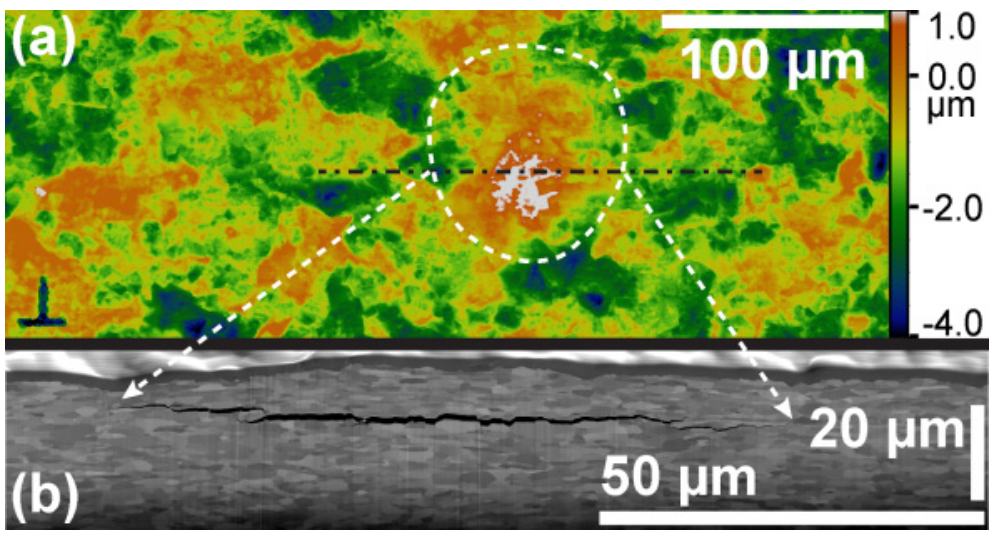

Figure 13: Blister on $W$ etched for 1 hour after D plasma exposure. This blister is located roughly in the center of Figure 8. Panel (a) shows the 3-D map of the exposed surface around the blister. The white dashed line indicates the circumference of the blister. The black dashdotted line indicates the location for the cross-section FIB cut. A " $T$ "'-marker is visible in the lower left corner of panel (a). Panel (b) shows a SEM micrograph of the cross-section prepared by FIB. Please note that the cross-section plane is tilted by $38^{\circ}$ with respect to the viewing plane.

The other conceivable influence of roughness lies in the surface area excess $\mathrm{X}_{\mathrm{A}}$. The ion flux from an ion beam or plasma source is, by definition, spread over the projected area of the sample. For our conditions with ion energies of tens of eV, the implantation depth of $\mathrm{D}$ ions is in the range of a few nanometers, i.e., far shallower than the depth of, e.g., blister cavities. This means that most of the implanted deuterons actually diffuse back to the surface, recombine with another $\mathrm{D}$ atom and desorb as $\mathrm{D}_{2}$ molecules instead of diffusing to a blister cavity and precipitating there as $\mathrm{D}_{2}$. Keeping in mind that the solubility of hydrogen isotopes in $\mathrm{W}$ is very low [17], this is easily understandable within Fick's diffusion theory: The concentration of solute D close to a surface is minimal (ideally zero), and maximal at the implantation range. In between, the solute D concentration decays linearly in steady state. As a consequence, the concentration gradient is steeper the closer a surface is to the implantation range, and as mentioned before, the closest surface is the implanted surface. Accordingly, the diffusive flux, which is proportional to the concentration gradient, is strongest towards this surface. Considering now that a sample with larger roughness, i.e., larger $\mathrm{X}_{\mathrm{A}}$, the surface area through which $\mathrm{D}$ can desorb also becomes larger. Thus, the balance of diffusive fluxes becomes even more biased towards re-emission rather than diffusion deeper into the sample. To illustrate this, we set up a simple 2-D diffusion model with a realistic surface that is derived from a high-resolution line scan across the W sample etched for 1 hour. We then deposit a number of $\mathrm{D}$ particles at a given distance below the sample surface and count how many particles are re-emitted through the front surface, and how many reach the back surface. The thickness of the simulation sample is 100 cells, the deepest point of the rough surface reaches to a depth of 40 cells. The width of the sample is 1260 cells. Mapping the simulation cells back to the actual surface scan, one cell corresponds to $100 \times 100 \mathrm{~nm}^{2}$, but in principle the simulation is independent of the actual scale, as long as the cells remain square. To calculate the increase of re-emission through the rough surface, we compare it to a sample with an 
ideally flat surface that has the same average thickness as the rough sample. This sample is 80 cells thick. The simulation results are visualized in Figure 14. For a shallow implantation only 1 simulation cell below the surface, the re-emission of the rough sample is $10.8 \%$ higher than for the flat sample. This value is identical to the excess length of the rough surface profile. For deeper implantation, the enhancement drops quickly towards zero, until for very deep implantation ( 50 cells below the surface) re-emission through the rough surface actually becomes smaller than for the flat one. The reason for this is that some particles are deposited very close to the back surface of the rough sample in this case. For illustration, the rough and flat surface profile, as well as the particle deposition profiles for very shallow (1 cell) and very deep (50 cells) implantation are plotted on the secondary y-axis of Figure 14. The quintessence of this simulation is that for rough samples with surface topographies as we investigate them here, the increase of re-emission with respect to smooth surfaces is small because the surface area excess is also small.

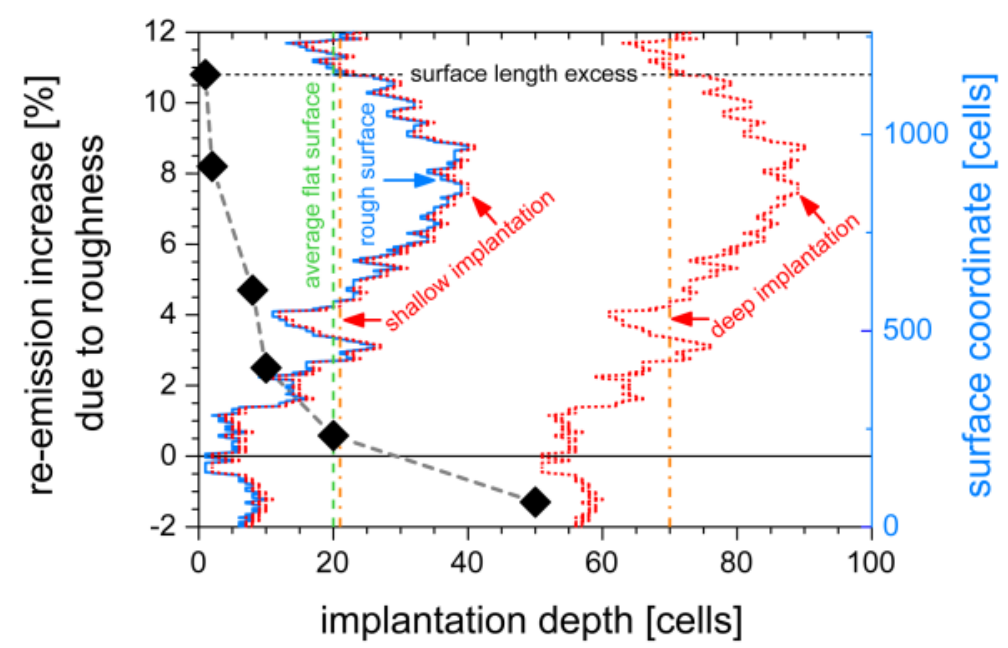

Figure 14: Estimation of re-emission from a rough surface by a 2-D diffusion simulation. The simulation grid consists of square cells with $100 \mathrm{~nm}$ side length, the back surface of the sample is located at a depth of 100 cells. The solid blue line represents a realistic surface profile extracted from the $W$ sample etched for 1 hour. The dashed green line indicates the position of a flat surface for a sample that has the same average thickness as the rough one. Dashed red lines indicate two implantation profiles into the rough sample with 1 cell ("shallow") and 50 cells ("deep") implantation. Orange dash-dotted lines show the same for the equivalent flat sample. The black diamonds (left y axis) indicate how much re-emission of particles through the front surface increases if that surface is not flat, but rough.

We note at this point that there are conceivable surface topographies where a network of deep but narrow cracks could occur, e.g., due to cyclic thermal overloading of surfaces by edgelocalised modes (ELMs) in a fusion reactor [15]. Such a surface is characterized by a relatively small $\mathrm{S}_{\mathrm{q}}$ but large $\mathrm{X}_{\mathrm{A}}$, which can actually be $>>1$. For example, if the average distance $\lambda$ between cracks is comparable to the average depth $\mathrm{d}$, and the crack width is negligible, $X_{A}=2 d / \lambda \approx 2$ (for the simplified case of a 2-D surface profile). For shallow 
implantation (i.e., into depths $<<$ d), such samples show very strong re-emission in an equivalent simulation as described above. Also, nano-scale roughness that we cannot resolve with the CLSM could lead to a substantial increase of $X_{\mathrm{A}}$. However, exemplary measurements using an atomic force microscope (AFM; Asylum Research MFP-3D-SA) did not indicate substantial nano-roughness or nano-cracks on our sample etched for 1 hour or on the technical surface. E.g., on a 30x30 $\mu \mathrm{m}$ scan area scanned with 1536x1536 data points ( $20 \mathrm{~nm} /$ point $)$, $\mathrm{X}_{\mathrm{A}}$ was nearly identical to the value derived from the CLSM measurement for the sample etched for 1 hour. For the technical surface, $X_{A}$ derived from such an AFM scan was about 2 times higher than the corresponding value derived from the CLSM measurement, i.e., still close to the value for the etched sample. However, the $30 \times 30 \mu \mathrm{m}$ scan area covered only one ridge and one groove, and is therefore not considered statistically relevant. Larger scans would have reduced the resolution due to a technical limitation of the image size. Smaller scans were investigated also, but are of course even less statistically relevant. The lateral resolution obtainable with the AFM tip used here was about 10-15 nm. This was estimated by measuring a few nm thick, non-continuous Au film on Si after performing the scans on the rough $\mathrm{W}$ samples. In addition, we also exemplarily investigated a metallographic crosssection of W with a technical surface using optical and scanning electron microscopy. This cross-section revealed that in some locations, crack systems that are connected to the surface and run more or less parallel to the surface exist. Such cracks are not observable by any of the surface diagnostics used here and thus do not contribute to the measured roughness parameters, but they would be one of the cases that could potentially strongly reduce D diffusion into depth. Judging from the investigated cross-section, these cracks seem to be few and far in between, so we assume that they are not a dominant factor in this specific case. However, for $\mathrm{W}$ with a technical surface finish obtained by a different process, such hidden crack systems may play a more crucial role.

Experimentally, the importance of $\mathrm{X}_{\mathrm{A}}$ for enhanced re-emission was recently shown by plasma-driven permeation experiments through plates of stainless steel that were machined into various model topographies [18]. Please note that for the material and exposure conditions investigated in [18], the boundary conditions for permeation were found to be recombination-limited on the plasma side of the sample and diffusion-limited on the permeation side, while in the case considered here, we assume diffusion-limited D reemission on all W surfaces (see also [19]).

In absolute numbers, the expected enhancement of re-emission due to roughness on the samples investigated here is of the order of $10 \%$ for the roughest ones. Since this is much smaller than the observed variations of blister coverage or volume, we currently consider reemission to be of minor importance and favor the explanation of surface fragmentation reducing the number of possible locations for blisters. However, we presently cannot exclude that the dependence of blistering on small variations of the effective $\mathrm{D}$ particle flux into the bulk is strongly non-linear in the parameter region investigated here.

Based on the discussion above, we finally come to the conclusion that roughness with values comparable to a technical surface has, overall, only a moderate effect on blistering. The much stronger reduction of blistering on a technical surface can therefore at present only be 
explained by the additional mechanical damage to the surface layer. Based on the currently available evidence, the relevant damage could be a strongly plastically deformed near-surface layer as well cracks introduced by the deformation, or, most likely, a combination of both. Indeed, it was already reported before that even on mirror-polished samples, the presence of a mechanical deformation layer close to the surface can strongly affect the occurrence and morphology of blisters on $\mathrm{W}$ due to $\mathrm{D}$ plasma exposure $[8,20]$.

\section{Summary}

In order to disentangle the effects of roughness and mechanical deformation of technical tungsten surfaces on blistering due to D plasma exposure, we prepared rough surfaces without a mechanical deformation layer by electropolishing and subsequent chemical etching for comparison to an actual technical W surface produced by grinding. We performed a detailed roughness analysis of all investigated surfaces. Based on that, we found that the surface area excess $X_{A}$, i.e., the fraction by which the actual 3-D surface area exceeds the projected area of a 3-D surface map, is a suitable parameter for describing the investigated surfaces. 2-D diffusion simulations also indicated that $\mathrm{X}_{\mathrm{A}}$ is closely linked to the increase of re-emission of implanted deuterium that is caused by the roughness. By acquiring 3-D difference maps of the same reference area before and after plasma exposure, we were able to doubtlessly show that, contrary to the prevalent opinion among researchers, blisters do occur on substantially roughened and even on technical surfaces, which contain mechanical damage in addition to roughness. We find that the influence of roughness alone on blistering is actually only moderate. We currently favor the explanation that this is mostly due to surface fragmentation, which limits possible locations for blister growth. Furthermore, we conclude from the comparison of a the technical surface with an etched surface with comparable roughness that the mechanical damage that is introduced by grinding to a technical surface finish reduces blistering much more strongly than the actual surface roughness.

\section{Acknowledgement}

This work has been carried out within the framework of the EUROfusion Consortium and has received funding from the Euratom research and training programme 2014-2018 under grant agreement No 633053. The views and opinions expressed herein do not necessarily reflect those of the European Commission. 


\section{References}

[1] J. Roth et al., J. Nucl. Mater. 390-391 (2009) 1-9

[2] M. Balden et al., J. Nucl. Mater. 414 (2011) 69-72

[3] R.D. Kolasinski et al., Phys. Scr. T138 (2009) 014042

[4] L. Gao et al., Nucl. Fusion 54 (2014) 122003

[5] M. Balden et al., J. Nucl. Mater. 452 (2014) 248-256

[6] M. Balden et al., J. Nucl. Mater. 438 (2013) S220-S223

[7] D. Nishijima et al., Nucl. Fusion 45 (2005) 669

[8] Y. Zayachuk et al., Nucl. Fusion 56 (2016) 086007

[9] A. Manhard, M. Balden, S. Elgeti, Pract. Metallogr. 52(8) (2015) 437-466

[10] A. Manhard, G. Matern, M. Balden, Pract. Metallogr. 50(1) (2013) 5-16

[11] A. Manhard, T. Schwarz-Selinger, W. Jacob, Plasma Sources Sci. Technol. 20 (2011) 015010

[12] S. Kapser, A. Manhard, U. von Toussaint, Nucl. Mater. Energy (2017), in press DOI: $10.1016 /$ j.nme.2016.11.019

[13] D. Nečas, P. Klapetek, Cent. Eur. J. Phys. 10 (2012) 181-8 (Gwyddion 2.45, released: 2016-04-26, http://gwyddion.net)

[14] M. Baldwin, R.P. Doerner, J. Nucl. Mater. 404 (2010) 165-173

[15] M. Wirtz et al., Phys. Scr. T145 (2011) 014058

[16] A. Manhard et al., Nucl. Mater. Energy (2016), in press, DOI: 10.1016/j.nme.2016.10.014

[17] R. Frauenfelder, J. Vac. Sci. Technol. 6 (1969) 388-397

[18] H. Zhou et al., J. Nucl. Mater. 463 (2015) 1066-1070

[19] R.A. Causey, J. Nucl. Mater. 300 (2002) 91-117

[20] S. Lindig et al., Phys. Scr. T145 (2011) 014039 